Document downloaded from:

http://hdl.handle.net/10251/104123

This paper must be cited as:

Pandal, A.; Pastor Enguídanos, JM.; Payri, R.; Kastengren, A.; Duke, DJ.; Matusik, KE.; Giraldo-Valderrama, JS.... (2017). Computational and Experimental Investigation of Interfacial Area in Near-Field Diesel Spray Simulation. SAE International Journal of Fuel and Lubricants. 10(2):1-9. doi:10.4271/2017-01-0859

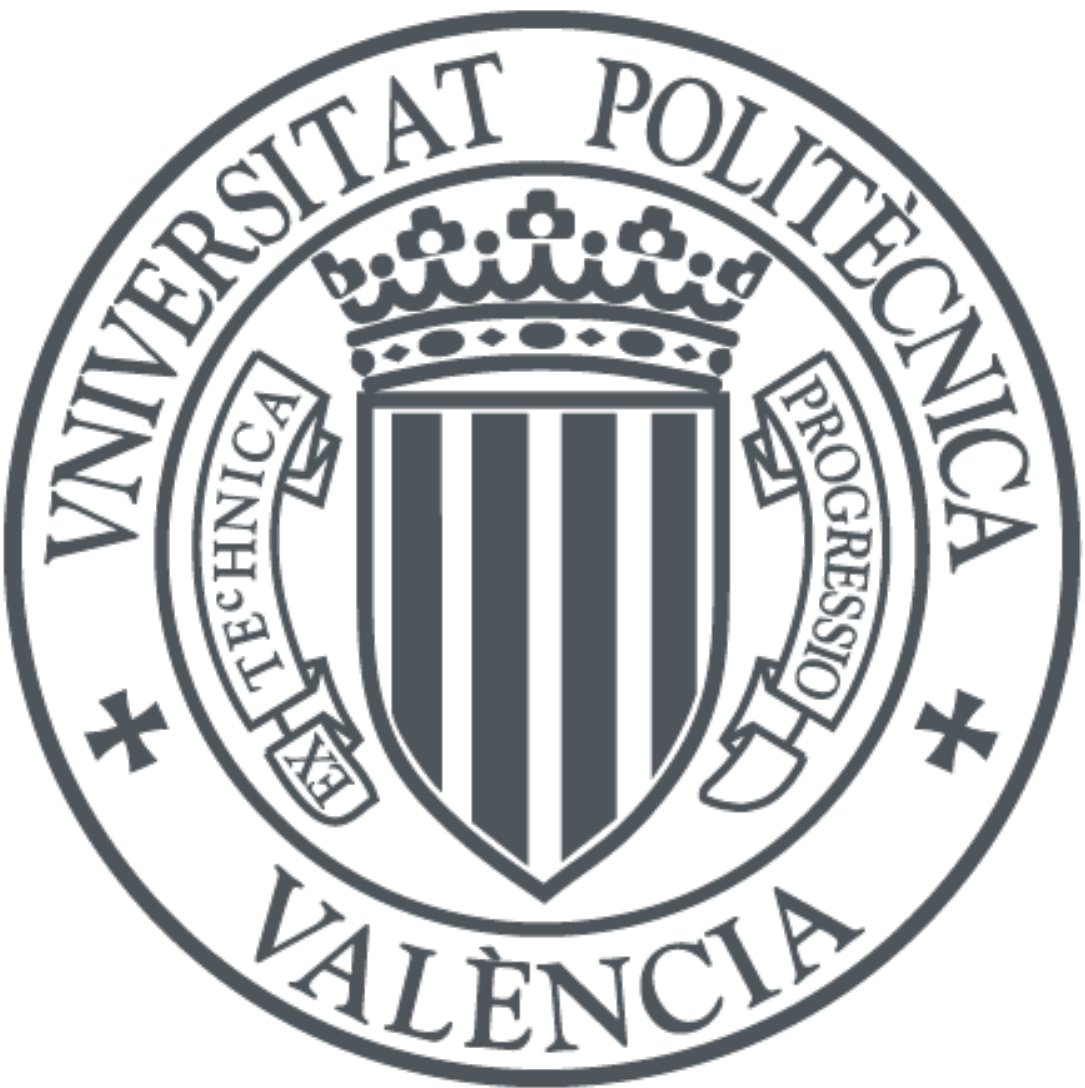

The final publication is available at

http://doi.org/10.4271/2017-01-0859

Copyright SAE International

Additional Information 


\title{
Computational and Experimental Investigation of Interfacial Area in Near-Field Diesel Spray Simulation
}

\author{
Author, co-author (Do NOT enter this information. It will be pulled from participant tab in \\ MyTechZone) \\ Affiliation (Do NOT enter this information. It will be pulled from participant tab in MyTechZone)
}

\begin{abstract}
The dense spray region in the near-field of diesel fuel injection remains an enigma. This region is difficult to interrogate with light in the visible range and difficult to model due to the rapid interaction between liquid and gas. In particular, modeling strategies that rely on Lagrangian particle tracking of droplets have struggled in this area. To better represent the strong interaction between phases, Eulerian modeling has proven particularly useful. Models built on the concept of surface area density are advantageous where primary and secondary atomization have not yet produced droplets, but rather form more complicated liquid structures. Surface area density, a more general concept than Lagrangian droplets, naturally represents liquid structures, no matter how complex. These surface area density models, however, have not been directly experimentally validated in the past due to the inability of optical methods to elucidate such a quantity. Optical diagnostics traditionally measure near-spherical droplet size far downstream, where the spray is optically thin. Using ultra-small-angle $\mathrm{x}$-ray scattering (USAXS) measurements to measure the surface area and $\mathrm{x}$-ray radiography to measure the density, we have been able to test one of the more speculative parts of Eulerian spray modeling. The modeling and experimental results have been combined to provide insight into near-field spray dynamics.
\end{abstract}

\section{Introduction}

Fuel injection transforms a high-speed liquid jet into a spray of fine droplets that quickly evaporate. Because of the extremely small length scales and high speeds, the details of the atomization process remain obscure. Both experimental and modeling investigations have suggested that the turbulent interfacial dynamics create a convoluted surface composed of a short jet core, with many wrinkles, ligaments, and droplets [1, 2].

Because the interface between the fuel and surrounding gas is so complex, it is an over-simplification to think of the dense spray core as being comprised of isolated droplets. Instead, we use the "more general notion" of interfacial area density [3]. This metric, with dimensions of reciprocal length, represents how much interfacial area is present per unit of volume.

With extreme levels of computational effort [4] the interface details can potentially be resolved through interface tracking. However, in a typical engineering calculation, the mesh resolution is considerably coarser than in these high fidelity computations. If one presumes that these interfacial details are far smaller than the mesh size, smoothing features over at least one cell, the end result is a diffuse-interface treatment in an Eulerian framework [5]. This framework is naturally extensible to near critical or super-critical regimes [6]. Rather than directly tracking the unresolved interface features, they are modeled in an Eulerian framework. Other Eulerian approaches such as multifluid 'size-of-classes' [7] have been also applied for Diesel spray simulations $[8,9]$, but still rely on the assumption of nearly spherical droplets.

Diffuse-interface Eulerian spray models can be used to predict the evolution of the interfacial density within the context of diesel fuel injection [10]. These models have two common elements: a model for the transport of liquid (or gas) and a model for the evolution of the interfacial surface area. The density of interfacial area is typically denoted by Sigma $(\Sigma)$ while the liquid fraction is denoted by Y. Hence, we refer to the strictly Eulerian model as a Sigma-Y approach, in contrast to ELSA (Eulerian-Lagrangian Spray Atomization), which includes a transition to Lagrangian particle tracking.

The transport of the liquid employs mass-averaged convection along with turbulent mixing. This model is derived from basic Favre averaging or LES filtering [11]. Thus, the accuracy of the liquid fraction transport is largely dependent on the accuracy of the twophase turbulent modeling. Despite the challenges of such modeling, there is at least an extensive theoretical basis to deal with the unclosed terms, putting the $\mathrm{Y}$ transport equation on a much firmer foundation that the interfacial evolution.

The interfacial evolution model, by predicting the amount of interfacial area per volume, can be used to predict drop size, once the primary atomization process is complete. This information is required if one wishes to employ a downstream transition to Lagrangian particle tracking, such as in the ELSA approach. However, the model for the interface evolution is somewhat more speculative, with several unclosed terms [12]. There are several interface modeling approaches that have been applied to sprays as researchers have explored competing ideas of how these terms should be treated [3, $11,14]$.

In recent years, models of the dense spray evolution have begun to favor Eulerian interfacial transport equations over Lagrangian particle tracking $[13,14]$. The advantages of Eulerian spray modeling in this region are as follows: 
1. Closer coupling between the transport of liquid mass and the other transport equations [15]

2. No need to assume discrete droplets in the densest parts of the spray [11]

3. With adequate mesh resolution, the Eulerian approach is less likely to be mesh dependent than Lagrangian modeling $[13,16]$

4. Good parallel scalability, without the necessity of handling load-balancing of Lagrangian particles [15]

For the present, Lagrangian particle tracking is the favored approach for in-cylinder simulations due to reasons of computational cost. However, with the diminishing cost of computational resources, Eulerian spray modeling has potential for applied engine development in the near future. Meanwhile, it offers valuable insights for studies of the near-field spray behavior and injector/spray coupling.

This paper uses a combined experimental and modeling approach to address the evolution of the interface in diesel fuel injection. The paper has several specific aims. The first is to validate the predictions of interfacial density more directly than previously possible. The interfacial density predictions have rarely been validated, and these validations have been in the context of downstream drop size. Using drop size for validation is not ideal, since we wish to avoid the assumption that the liquid is in the form of droplets. A few prior examples used DNS simulations [17] for validation. Others have emphasized the location of the mass, but not the interfacial density [10]. If possible, we wish to validate directly versus experiments without resorting to the assumption of spherical droplets. This can be done via USAXS measurements, as explained in the next section.

It has been postulated that diesel spray evolution is dominated by turbulent dispersion rather than interfacial dynamics [18]. This mixing-limited hypothesis asserts that the details of the interface are irrelevant under diesel-relevant conditions, such as high ambient gas density and high injection velocity. In support of this hypothesis Garcia-Oliver et al. [10] noted successful prediction of numerous spray metrics at high ambient gas density conditions, with diminishing accuracy at lower gas densities.

There is no feedback mechanism in the typical Eulerian model by which the interfacial dynamics can impact the transport of the liquid fraction. Hence, by its current structure, the Sigma-Y model assumes that the mixing-limited conditions apply. In the current work, we study the spray under high ambient pressure conditions but low ambient temperature, unlike the evaporating conditions studied previously. We thus further investigate the range of applicability of this modeling framework.

In summary, we wish to (1) validate the transport of liquid mass and (2) assess when the model for interfacial evolution is accurate.

\section{Experimental Methodology}

The goal was to measure interfacial surface area in the dense spray core, near the injector exit, where the assumption of individual droplets may not be valid. Ultra-small angle $x$-ray scattering (USAXS) was chosen as a diagnostic technique due to its ability to interrogate the dense region and provide quantitative information about the complex interface without resorting to the assumption that the liquid is in the form of droplets [16].
USAXS measurements were performed at the 9-ID beamline of the Advanced Photon Source at Argonne National Laboratory. A solenoid diesel injection nozzle provided by the Engine Combustion Network (ECN) was investigated; the single-hole Spray A 210675 nozzle with a nominal hole diameter of $90 \mu \mathrm{m}$. The injector was mounted horizontally in a $0.5 \mathrm{~L}$ vessel pressurized with $\mathrm{N}_{2}$ gas, which also supplied a purge flow of approximately 4 standard $\mathrm{L} \mathrm{min}{ }^{-1}$ in order to inhibit droplet accumulation within the domain during measurements. Table 1 summarizes the measured rail and chamber pressure combinations for the injection nozzle.

Table 1.List of investigated USAXS pressure conditions for each diesel injection nozzle.

\begin{tabular}{|l|l|l|l|l|}
\hline Injector & \multicolumn{2}{|l|}{ Rail Pressure (bar) } & Chamber Pressure (bar) \\
\hline Spray A 210675 & \multicolumn{2}{|l|}{1500} & 6.7 \\
\hline Spray A 210675 & 1500 & 1000 & 500 & 20 \\
\hline
\end{tabular}

A diesel common-rail injection system was used to pressurize ndodecane fuel to the desired rail pressure. Table 2 lists the fuel properties at room temperature and pressure conditions.

Table 2.Properties of n-dodecane at $25^{\circ} \mathrm{C}$ and 1 bar.

\begin{tabular}{|l|l|l|}
\hline Fuel Property & Value & Units \\
\hline Density $(\rho)$ & 746 & $\mathrm{~kg} \mathrm{~m}^{-3}$ \\
\hline Viscosity $(\mu)$ & $1.8 \times 10^{-6}$ & $\mathrm{~m}^{2} \mathrm{~s}^{-1}$ \\
\hline Surface Tension $(\sigma)$ & 0.025 & $\mathrm{~N} \mathrm{~m}^{-1}$ \\
\hline
\end{tabular}

The injection temperature was approximately 60,40 , and $30^{\circ} \mathrm{C}$ at 1500, 1000, and 500 bar, respectively. Each injector was fired at $3 \mathrm{~Hz}$ with an injection duration of approximately $2.5 \mathrm{~ms}$. USAXS data were recorded in a $1 \mathrm{~ms}$ interval during the steady-state portion of the spray. Background measurements were also recorded over $80 \mathrm{~ms}$ before each scan to account for any changes within the measurement domain caused by previous spray events.

The Bonse-Hart instrument at the 9-IDbeamline measures the scattering intensity, $\mathrm{I}_{\text {scat }}(q)$, for a wide range of scattering vectors, $q$ [20]. A beam of $x$-rays at $17.9 \mathrm{keV}$ is first shaped into a $100 \times 500$ $\mu \mathrm{m} \mathrm{H} \times \mathrm{V}$ spot with a set of high precision $2 \mathrm{D}$ slits. The incoming beam is collimated with a pair of Si (220) crystals before impinging on the spray, from which the incident x-rays scatter at small angles. The scattered x-rays are filtered downstream with a pair of Si (220) analyzer crystals, and the scattered photons are measured with a detector. The pair of analyzer crystals is rotated to measure the transmitted beam intensity as a function of scattering vector. The scattering vector was varied between $1 \times 10^{-4} \AA^{-1}<q<1 \times 10^{-2} \AA^{-1}$ with a step size of $1 \times 10^{-5} \AA^{-1}$ at low $q$, with increasing step size for larger $q$. The scattering intensity as a function of $q$ was measured at axial distances ranging from 1 to $20 \mathrm{~mm}$ downstream of the injection nozzle, at the centerline of the spray. Once $\mathrm{I}_{\text {scat }}(q)$ is known, the differential cross-section may be calculated, and related to the total shape and surface area per volume of fuel droplets, with postprocessing performed using the Irena data analysis package [21]. 


\section{Modeling Approach}

The Sigma-Y model considers the liquid/gas mixture as a pseudofluid with a single velocity field. Considering that the flow exiting the injector is operating at large Reynolds and Weber numbers, it is possible to assume a separation of the large scale flow features, such as mass transport, from the atomization process occurring at smaller scales. This allows the direct simulation of the large scale bulk transport of the liquid while unresolved turbulent transport is modeled using standard closures such as those used in Reynoldsaveraged turbulence models.

To track the dispersion of the liquid phase an indicator function is used, taking a value of unity in the liquid phase and zero in the gas phase. The mean liquid volume fraction is denoted $(\overline{\boldsymbol{Y}})$ and the mean mass averaged fraction is defined as $(\widetilde{\boldsymbol{Y}}=\overline{\boldsymbol{\rho} \boldsymbol{Y}} / \overline{\boldsymbol{\rho}})$. Favre averagingthe transport equation for the liquid mass fraction yields Eq. (1)

$$
\frac{\partial \bar{\rho} \widetilde{Y}}{\partial t}+\frac{\partial \bar{\rho} \widetilde{u_{i}} \widetilde{Y}}{\partial x_{i}}=-\frac{\partial \bar{\rho} \widetilde{u_{i} Y}}{\partial x_{i}}
$$

Where $\boldsymbol{u}^{\prime}$ denotes the density weighted turbulent fluctuations in velocity and $\boldsymbol{Y}^{\prime}$ denotes turbulent fluctuations in liquid mass fraction. The turbulent diffusion liquid flux term, $\widetilde{\boldsymbol{u}_{i} \boldsymbol{Y}}$, captures the effect of the relative velocity between the two phases [22]. This term is modeled using a standard turbulent gradient flux model, which successfully worked for Diesel spray compared to DNS results, as indicated in [12].

$\bar{\rho} \widetilde{u_{i} Y}=-\frac{\mu_{t}}{S_{c}} \frac{\partial \widetilde{Y}}{\partial x_{i}}$

Where $\boldsymbol{\mu}_{\boldsymbol{t}}$ is the turbulent viscosity and Sc the Schmidt number.

While the approach used here assumes that the resolved momentum of the liquid/gas mixture can be characterized by a single bulk velocity, the slip velocity can be expressed explicitly as derived by [11] and seen in Eq. (3)

$u_{i_{l}}-u_{i_{g}}=\frac{1}{\widetilde{Y}(1-\widetilde{Y})} \widetilde{u_{i} Y}$

Under the assumption that the two phases form an immiscible mixture, the mass-averaged value of the indicator function is related to the density by:

$\frac{1}{\bar{\rho}}=\frac{\widetilde{Y}}{\rho_{l}}+\frac{1-\widetilde{Y}}{\rho_{g}}$

An equation of state is then assigned to each phase. The gas phase obeys an ideal gas law, while the liquid phase is estimated following the Hankinson-Brobst-Thomson (HBT) correlation [23], in which the liquid density is a function of temperature (T) and pressure (p).

To close the above system of equations, the temperature is obtained from a bulk mixture enthalpy equation expressed in the following terms:
$h(T)=\widetilde{Y} \cdot h_{l}(T)+(1-\widetilde{Y}) \cdot h_{g}(T)$

Here $\boldsymbol{h}_{\boldsymbol{l}}$ and $\boldsymbol{h}_{\boldsymbol{g}}$ denote the enthalpy of the liquid and gas phases respectively, and are calculated as the integrals of

$d h_{l}(T)=c_{p, l} d T$

$d h_{g}(T)=c_{p, g} d T$

Where $\boldsymbol{c}_{\boldsymbol{p}, \boldsymbol{i}}$ is the specific heat capacity at constant pressure. This is obtained as a function of temperature ( $\mathrm{T})$ from a set of coefficients taken from JANAF tables of thermodynamics. This can be directly applied to the ambient gas, but not for the fuel. In this case, upon the principle of corresponding states, the Rowlinson-Bondi equation [23] that makes use of the departure heat capacity function is applied.

Finally, being $h$ the static enthalpy implemented through the following conservation equation, where $\boldsymbol{\alpha}_{\boldsymbol{e f f}}$ is the effective turbulent thermal diffusivity and $\boldsymbol{\tau}_{\boldsymbol{i} j} \frac{\boldsymbol{\partial} \boldsymbol{u}_{\boldsymbol{j}}}{\partial \boldsymbol{x}_{\boldsymbol{i}}}$ the viscous dissipation:

$\frac{\partial \bar{\rho} h}{\partial t}+\frac{\partial \bar{\rho} \widetilde{u_{i}} h}{\partial x_{i}}-\frac{\partial}{\partial x_{j}}\left(\alpha_{e f f} \frac{\partial h}{\partial x_{i}}\right)=\frac{\partial p}{\partial t}+u_{i} \frac{\partial p}{\partial x_{i}}+\tau_{i j} \frac{\partial u_{j}}{\partial x_{i}}$

The solution of the above equations fully characterizes the large-scale bulk motion of the flow. Several other options exist for obtaining closure in the above system of equations (see for example the discussion in [11] and [24].

Conversely, the small scale atomization is modeled by solving a transport equation for the evolution of the interphase surface area density Sigma. This surface density can be understood as the amount of spatial surface per unit volume at a given time and spatial position. It is not easy to establish even an unclosed form of the balance equation of this quantity. Ishii [25] and Delhaye et al. [26] made some attempts on two phase flow applications and by means of the spatial averaging operator, the averaged surface density equation is introduced by Ishii [25] in a similar form to the development of flame surface area density $[27,28]$ used in combustion applications. Then, Ishii's original equation was adopted by Vallet and Borghi [29], in which nearly all the models in the literature are based, and gives the following evolution equation for this quantity, assuming a gradient law closure for the turbulent diffusion flux term, where $\boldsymbol{D}_{\boldsymbol{\Sigma}}$ is a suitable diffusion coefficient usually taken as the turbulent kinematic viscosity $\left(\boldsymbol{v}_{\boldsymbol{t}}\right)$ over a Schmidt number $\left(\boldsymbol{S}_{\boldsymbol{c}_{\boldsymbol{\Sigma}}}\right)$.

$\frac{\partial \widetilde{\Sigma}}{\partial t}+\frac{\partial \widetilde{u_{j}} \widetilde{\Sigma}}{\partial x_{j}}-\frac{\partial}{\partial x_{j}}\left(D_{\Sigma} \frac{\partial \widetilde{\Sigma}}{\partial x_{j}}\right)-a \widetilde{\Sigma}+b \widetilde{\Sigma}^{2}-S_{\Sigma_{\text {init }}}=0$

where the inverse time-scale $a$ and coefficient $b$ can be understood as the surface generation due to the growth of fluid instabilities (i.e. Kelvin-Helmholtz) and the destruction of surface due to droplet coalescence (in the case of dispersed flow), respectively. However, the most common form for the combination of these two source terms is the restoration to an equilibrium value $\left(\overline{\boldsymbol{\Sigma}}_{\boldsymbol{e q}}\right)[19,30]$ :

$\frac{\partial \widetilde{\Sigma}}{\partial t}+\frac{\partial \widetilde{u_{j}} \widetilde{\Sigma}}{\partial x_{j}}-\frac{\partial}{\partial x_{j}}\left(D_{\Sigma} \frac{\partial \widetilde{\Sigma}}{\partial x_{j}}\right)-C_{\Sigma} \widetilde{\Sigma}\left(1-\frac{\widetilde{\Sigma}}{\bar{\Sigma}_{e q}}\right)-S_{\Sigma_{\text {init }}}$
$=0$ 
The $\overline{\boldsymbol{\Sigma}}_{\boldsymbol{e q}}$, already mentioned, is the equilibrium or critical surface density to which the local surface density is driven and it is set by a suitable equilibrium droplet radius $\left(\boldsymbol{r}_{\boldsymbol{e q}}\right)$ :

$\bar{\Sigma}_{e q}=\frac{3 \bar{\rho} \widetilde{Y}}{\rho_{l} r_{e q}}$

As proposed by Vallet et al. [22], assuming that droplet collision is the principal mechanism in the droplet breakup and the kinetic energy of colliding droplets equals the minimum increase of the surface tension energy at the break-up, the equilibrium radius can be derived:

$r_{e q}=\alpha_{2} \frac{\sigma^{3 / 5} l_{t}^{2 / 5}}{\widetilde{k}^{3 / 5}} \frac{(\bar{\rho} \widetilde{Y})^{2 / 15}}{\rho_{l}^{11 / 15}}$

Then, the coefficient $\boldsymbol{C}_{\boldsymbol{\Sigma}}$ is modeled as the inverse of the turbulent time scale:

$C_{\Sigma}=\alpha_{1} \frac{\tilde{\varepsilon}}{\widetilde{\boldsymbol{k}}}$

Note the presence of two modeling constants $\left(\boldsymbol{\alpha}_{1}, \boldsymbol{\alpha}_{2}\right)$, which by default are equal to 1 [22], although other values have been evaluated $[19,27,31,32]$.

Finally, as can be seen all the source terms that are involved in this equation are proportional to the interface surface density Sigma. As a result, there will be no production if there is no interface. Therefore, a proper initialization should be made by means of the term $\boldsymbol{S}_{\boldsymbol{\Sigma}_{\text {init }}}$. For that purpose, a minimum value of $\boldsymbol{\Sigma}$ is considered in any computational cell which is not filled with pure liquid or gas. In a similar way as in Wang et al. [33], this minimum value is estimated as $V^{-1 / 3}$ where $\mathrm{V}$ is the volume of the CFD cell. The source term only takes a positive value if the interface field is lower than this $\Sigma_{\text {min }}$ :

$\Sigma_{\text {init }}=\frac{\Sigma_{\min }-\Sigma}{\Delta t} \operatorname{pos}\left(\Sigma_{\min }-\Sigma\right)$

Where pos() is a Boolean pre-implemented operator in OpenFOAM:

$\operatorname{pos}(x)=\left\{\begin{array}{l}1 \text { if } x>0 \\ 0 \text { if } x \leq 0\end{array}\right.$

Together with the mass averaged liquid fraction, the interphase surface area density can be used to derive results for droplet sizing, such as the local SMD $\left(\boldsymbol{D}_{\mathbf{3 2}}\right)$ of the spray and the drop number density.

$D_{32}=\frac{6 \bar{\rho} \widetilde{Y}}{\rho_{l} \widetilde{\Sigma}}$

$n=\frac{\rho_{l}^{2} \widetilde{\Sigma}^{3}}{36 \pi \bar{\rho}^{2} \widetilde{Y}^{2}}$

A further description of the numerical implementation of this solver can be found in [10].

\section{Computational Model Setup}

To simulate the diesel sprays with the $\Sigma$-Y model, a 2-D axisymmetric computational domain, with $80 \mathrm{~mm}$ in length and $25 \mathrm{~mm}$ in radius, is used (note that OpenFOAM requires a 3-D wedge grid in order to perform 2-D axisymmetric calculations). The mesh is structured with non-uniform grid resolution. There are 20 cells along the orifice diameter, keeping an aspect ratio close to one in the near nozzle region, as depicted in Figure 1. The non-uniform grid resolution consists of cells with an expansion ratio of 1.01 and 1.06 in the axial and radial directions, respectively, as indicated in [10]. This mesh construction is the result of different sensitivity studies previously performed, including an evaluation of nozzle resolution [12].

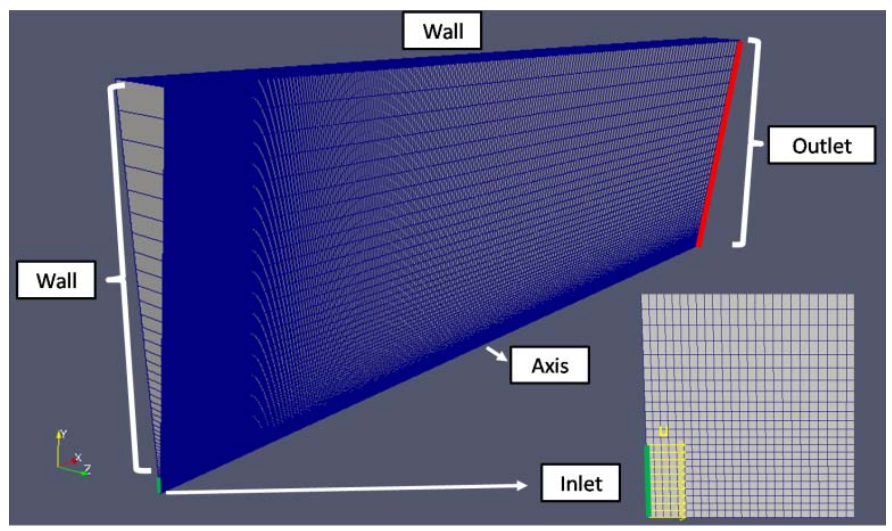

Figure 1. Computational domain used for CFD simulations.

Boundary conditions selected for all the walls of the domains are noslip while for both side planes, the symmetry boundary condition is chosen. A non-reflective boundary condition is used for the outlet, and for the inlet boundary condition; a mapping procedure is conducted in order to feed the simulation with the fields obtained at the nozzle exit in a coupled computational domain (including injector nozzle geometry), as explained in [36]. To do that, the mapped boundary condition of OpenFOAM is used.

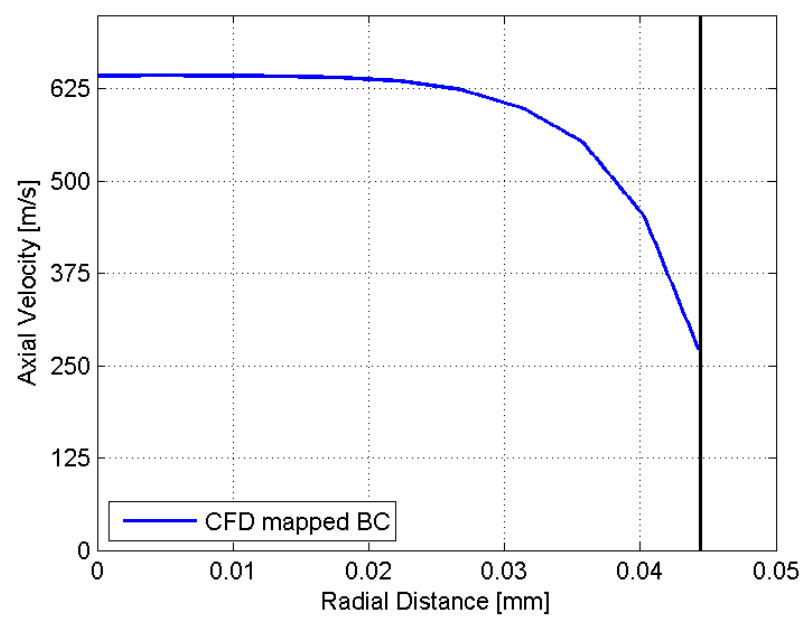

Figure 2. Computed axial velocity profile at $1 \mathrm{~ms}$ after SOI at the orifice for mapped inlet boundary condition simulation. Black solid line depicts the radius of the nozzle orifice. 
In order to clarify this configuration, in Figure 2 the axial velocity profile at the nozzle orifice is shown (note that only one half of the profile is depicted due to the axisymmetric computational domain). One can observe that using this boundary condition, the effects derived from the in-nozzle flow can be included in the external flow simulation. Together with the velocity field, the density and the turbulence quantities (see Figure 3), i.e. turbulent kinetic energy (k) and turbulent dissipation $(\varepsilon)$, are also imposed at the inlet boundary condition using the mapping procedure.
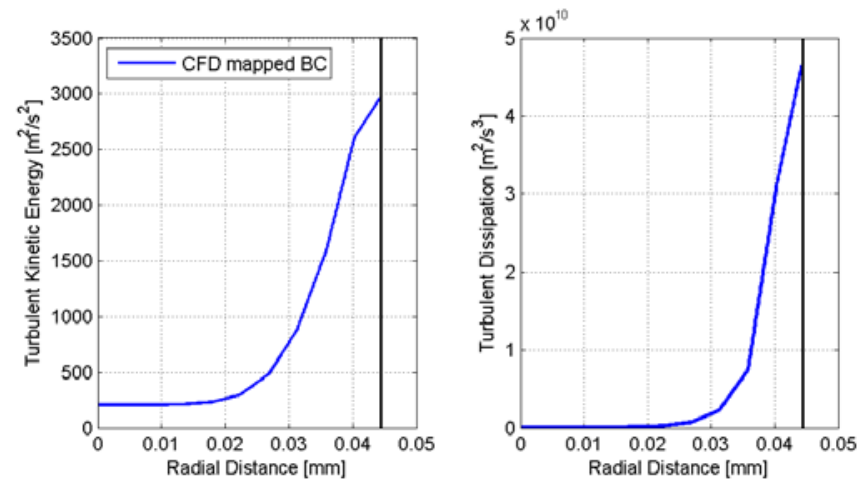

Figure 3. TKE [left] and turbulence dissipation [right] at 1ms after SOI at the orifice for mapped inlet boundary condition simulation. Black solid line depicts the radius of the nozzle orifice.
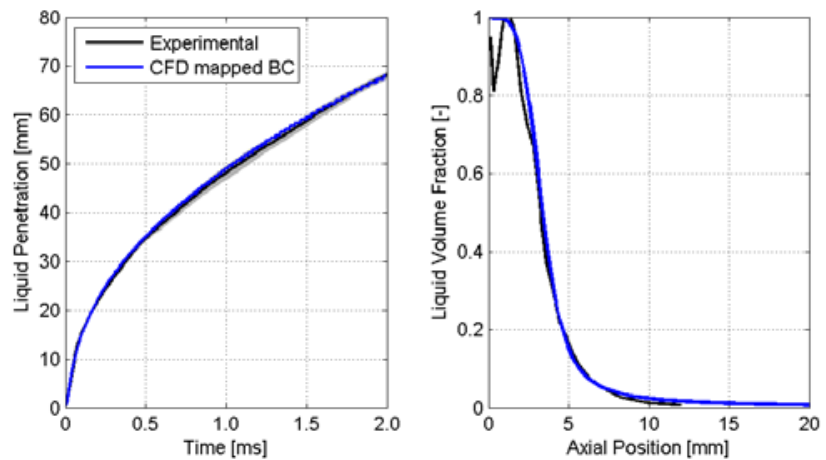

Figure 4. Spray penetration [left] and computed centerline liquid volume fraction at $1 \mathrm{~ms}$ after SOI [right] for mapped inlet boundary condition simulation.
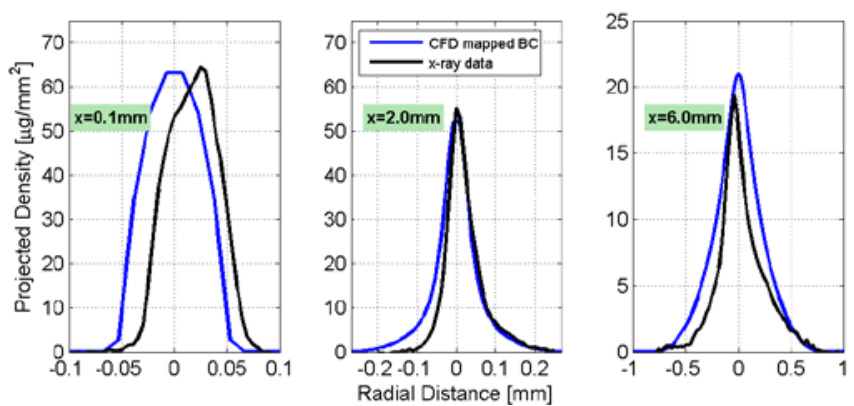

Figure 5. Computed and measured profiles of projected mass density [ $\mu \mathrm{g} / \mathrm{mm} 2]$ at $500 \mu \mathrm{s}$ after SOI at axial locations of $0.1 \mathrm{~mm}, 2 \mathrm{~mm}$ and $6 \mathrm{~mm}$ downstream the nozzle exit for mapped inlet boundary condition simulation.

The k- $\varepsilon$ turbulence model was set to perform the simulations, as seen previously, but due to the well-known round jet spreading overprediction of k- $\varepsilon$ type models [34], a corrected value (1.60) for $C_{1 \varepsilon}$ is Page 5 of 9 used, as indicated in [10]. Pope [34] has previously suggested that the latter value should be used for round jets. The liquid turbulent flux closure [35] is calculated by means of a gradient closure, the discretization of the divergence terms was solved with a Gamma NVD scheme, and a first order Euler scheme is applied for time derivative terms.

As presented in Desantes et al. [36], with this kind of configuration it is possible to closely match typical global spray parameters such as penetration and liquid volume fraction (LVF), with excellent prediction of the intact liquid core $(\mathrm{LVF}>0.9)$, which can be seen in Figure 4, as well as other specific parameters used to describe the internal structure of a diesel spray, such as the projected density (see Figure 5) or the transverse integrated mass from x-ray measurements. Thus, it is a suitable configuration to include nozzle effects in the simulation while keeping the computational effort reduced.

\section{Results}

Because the previous work of Desantes et al. [36] already confirmed the ability of the model to predict more traditional spray metrics, such as penetration and mass distribution, the present work evaluates the model's ability to predict interfacial surface evolution. In order to validate the interfacial predictions most directly, modeling predictions are compared against experimental measurements of surface area made using the USAXS technique [37]. As explained in [37], the provided experimental surface area is likewise line-of-sight integrated so CFD predictions must be processed to allow a fair comparison. The interface surface density $(\Sigma)$ value within each CFD cell is integrated through the depth of the spray, collapsing the surface area to a 2-D map (it should be noted that for 2D computations, axisymmetry is assumed).

Concerning the configuration of the interface surface density equation, the default values of the modeling constants (see Reference case in Table 3) are first selected to conduct an initial evaluation of the performance achieved by the model. To do that, among all the experimental conditions available, the one corresponding to the intermediate injection pressure $\left(\mathrm{P}_{\mathrm{inj}}=100 \mathrm{MPa}\right)$ is chosen.
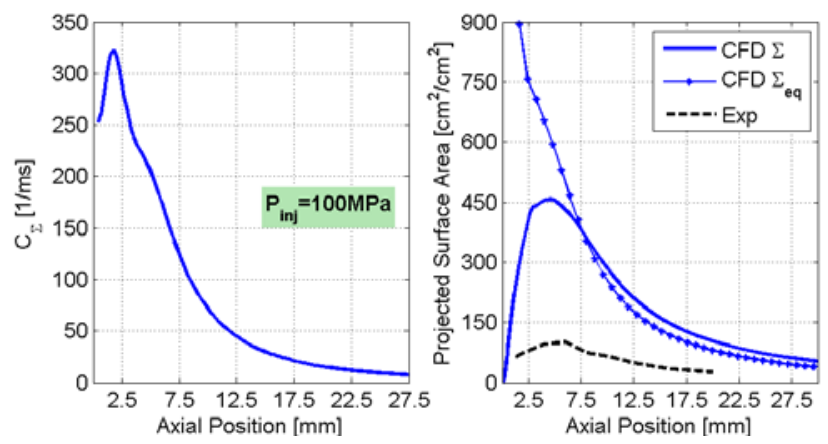

Figure 6. $\mathrm{C}_{\Sigma}$ coefficient [left] and projected surface area [right] results at $1.5 \mathrm{~ms}$ after SOI for the default values of the modeling constants. $\mathrm{P}_{\text {inj }}=100$ $\mathrm{MPa}, \rho_{\mathrm{amb}}=22.8 \mathrm{~kg} / \mathrm{m} 3$

In Figure 6, the two $\Sigma$ equation parameters $\left(\mathrm{C}_{\Sigma}\right.$ and $\left.\Sigma_{\text {eq }}\right)$ controlled by the modeling constants, $\alpha_{1}$ and $\alpha_{2}$, are depicted. On the left image the axial variation of the time scale, $C_{\Sigma}$ is shown, which indicates how fast the predicted interfacial area density $(\Sigma)$ evolves towards the equilibrium value $\left(\Sigma_{\text {eq }}\right)$. On the right graph both the predicted (blue solid line) and measured (black dashed line) projected surface area and also the predicted equilibrium value (blue pointed line), are 
plotted. At the sight of the results, it can be stated that the default configuration for $\alpha_{1}$ and $\alpha_{2}$ over-predicts the surface area results and also that a lower equilibrium value is probably required to be able to match these measurements.

Then, it was decided to conduct a parametric study in order to investigate the effects of the constant values individually on the results. In Table 3 the different simulations proposed are presented, indicating the respective values adopted by each modeling constant.

Table 3. Parametric studies conducted for the interphase surface density equation setup

\begin{tabular}{|l|l|l|}
\hline Case & $\mathbf{\alpha}_{1}[-]$ & $\mathbf{\alpha}_{2}[-]$ \\
\hline Reference & 1.0 & 1.0 \\
\hline Alpha1 Low & $\mathbf{0 . 3}$ & 1.0 \\
\hline Alpha1 Medium & $\mathbf{0 . 7}$ & 1.0 \\
\hline Alpha1 High & $\mathbf{1 . 5}$ & 1.0 \\
\hline Alpha2 Low & 1.0 & $\mathbf{0 . 7}$ \\
\hline Alpha2 Medium & 1.0 & $\mathbf{2 . 0}$ \\
\hline Alpha2 High & 1.0 & $\mathbf{3 . 5}$ \\
\hline
\end{tabular}

According to the previous table, the first investigation conducted considers the $\alpha_{1}$ constant. In Figure 7 , together with the reference case, different constant values below and over the reference are evaluated. Once again, apart from the projected surface area, the two parameters $\mathrm{C}_{\Sigma}$ and $\Sigma_{\text {eq }}$ are depicted. Here, it can be perfectly observed the impact of the first modeling constant which smoothly drives the CFD Sigma towards the equilibrium value (note that this value is the same for all the configurations evaluated). The results indicate that a higher $\boldsymbol{\alpha}_{1}$ value makes the transition faster and vice versa. Concerning the suitable configuration, it seems that a value lower than one (in the vicinity of 0.7 ) is needed to give some curvature to the predicted profile at the beginning. However, this value must be higher than 0.3 which distorts too much the shape of the projected surface area. In any manner, these simulations depict that a better prediction of the equilibrium surface area, i.e. closer to the experimental measurement, is the key factor to provide good CFD modeling performance.
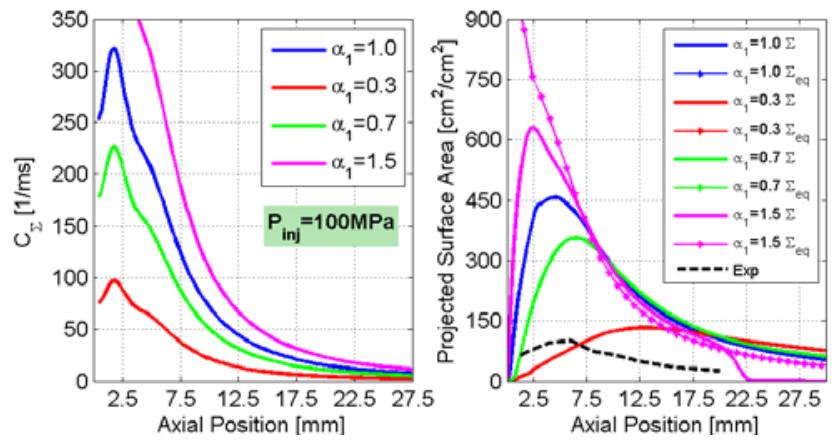

Figure 7. $C_{\Sigma}$ coefficient [left] and projected surface area [right] results at $1.5 \mathrm{~ms}$ after SOI for the parametric study of $\alpha_{1}$ constant. $\mathrm{P}_{\text {inj }}=100 \mathrm{MPa}, \rho_{\mathrm{amb}}=$ $22.8 \mathrm{~kg} / \mathrm{m}^{3}$.

Thus, the investigation of the second modeling constant is considered in Figure 8.In this case, all the simulations tend to the Sigma equilibrium value with the same pace (Figure 8[left]) but quite different surface area predictions are achieved. The surface area predicted axial profile is higher when the constant $\alpha_{2}$ is reduced while increasing its value, progressively drives the solution to a lower profile. These predictions suggest that a suitable $\alpha_{2}$ constant should take a value close to 3 .

After the parametric studies, some possible optimum parameter combinations are evaluated in order to finally been able to choose a proper setup. In Table 4 the investigated combinations are collected, showing the respective values adopted by each modeling constant.
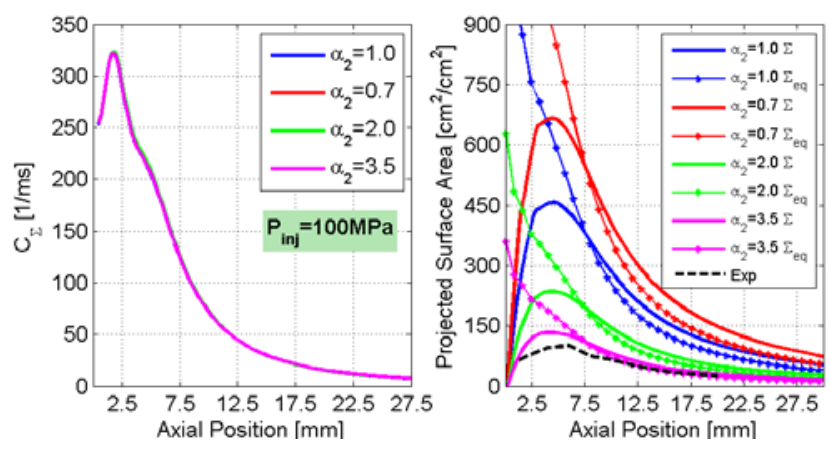

Figure 8. $\mathrm{C}_{\Sigma}$ coefficient [left] and projected surface area [right] results at $1.5 \mathrm{~ms}$ after SOI for the parametric study of $\alpha_{2}$ constant. $\mathrm{P}_{\mathrm{inj}}=100 \mathrm{MPa}, \rho_{\mathrm{amb}}=$ $22.8 \mathrm{~kg} / \mathrm{m}^{3}$.

Table 4. Combination studies conducted for the interphase surface density equation setup

\begin{tabular}{|l|l|l|}
\hline Case & $\mathbf{\alpha}_{1}[-]$ & $\mathbf{\alpha}_{2}[-]$ \\
\hline Comb 1 & 0.5 & 2.5 \\
\hline Comb 2 & 0.7 & 3.0 \\
\hline Comb 3 & 0.8 & 3.5 \\
\hline Comb 4 & 0.8 & 4.0 \\
\hline
\end{tabular}

In Figure 9, the results achieved with these combinations are presented. Unlike the previous figures, in this case only the predicted interfacial area density $(\Sigma)$ is plotted at the right side against the experimental measurement. In general, predictions are mainly controlled by the $\alpha_{2}$ parameter. It seems that a value lower than 3.5 overestimates the interphase density, in comparison with the experiments, while a higher value (e.g. $\alpha_{2}=4.0$ ) underpredicts the surface formation between the liquid and the gas. Regarding the $\alpha_{1}$, as previously mentioned, a value lower than one able to give some curvature to the profile is needed, with this comparison the value of 0.8 appears to be appropriate. In light of the predictions, with $\alpha_{1}=0.8$ and $\alpha_{2}=3.5$ the CFD simulation is able to match the measurements with great deal of accuracy and as a result, it is chosen as the optimum for the following calculations. 

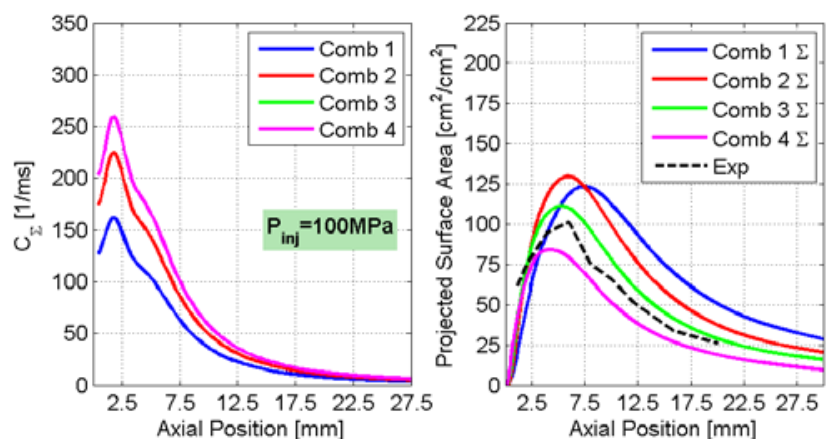

Figure 9. $\mathrm{C}_{\Sigma}$ coefficient [left] and projected surface area [right] results at $1.5 \mathrm{~ms}$ after SOI for different parameter combinations. $\mathrm{P}_{\text {inj }}=100 \mathrm{MPa}, \rho_{\text {amb }}=$ $22.8 \mathrm{~kg} / \mathrm{m} 3$.

\section{Optimum Configuration}

In the following, the results for the optimum chosen setup are depicted again at $1.5 \mathrm{~ms}$ after SOI. In Figure 10, the influence of injection pressure is shown. Experimental trends are well reproduced; decreased injection pressure decreases the surface area profile, as well as the location at which the maximum occurs, with little changes with injection pressure. Predictions of this set of optimum parameters are remarkably close to the experimental data, providing a clear improvement with respect to the default values.

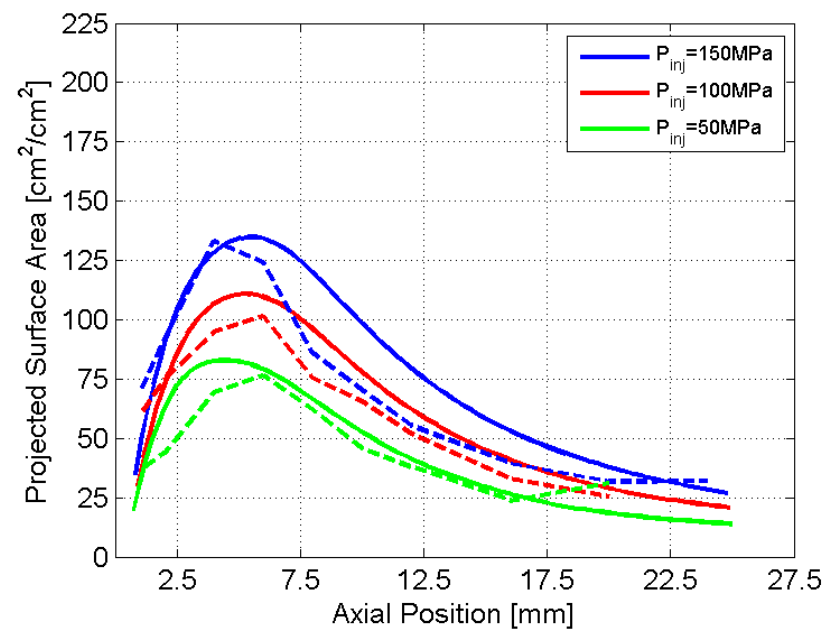

Figure 10. Computed (solid lines) and measured (dashed lines) projected surface area profiles for different injection pressures

Finally, the influence of the back pressure, i.e. ambient density, is shown in Figure 11. Experimental trends are well reproduced; decreased back pressure shows a lower increase of the interphase surface as well as a smoother and slower decrease of the profile with the axial distance, which means a lower atomization rate. These quite great predictions confirm that the chosen set of values for the interphase surface density equation is a suitable one. There is some reduction of accuracy only at the lowest downstream pressure, where the assumption of mixing-limited transport may not be as appropriate.

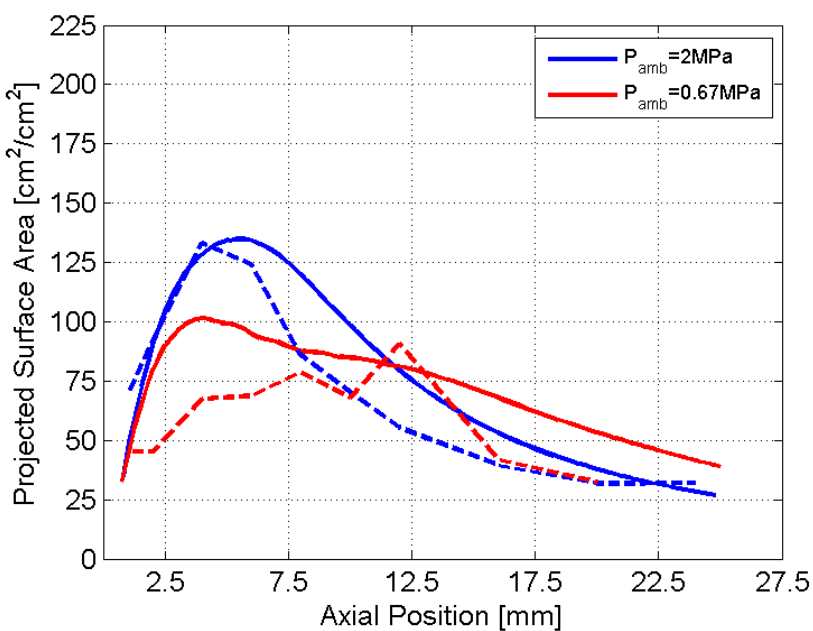

Figure 11. Computed (solid lines) and measured (dashed lines) projected surface area profiles for different back pressures

Additionally, the CFD model provides Sauter Mean Diameter (SMD) predictions derived from the Sigma and LVF values, which is a spray metric easy to evaluate. Thus, in Figure 12, computed SMD axial profiles for the three injection pressure conditions are depicted from $2.5 \mathrm{~mm}$ downstream, i.e. only considered downstream of the intact liquid core. The trends seem to be quite reasonable: increased injection pressure decreases the droplet size and vice versa. Predictions depict that after a first atomization region, an increase with axial distance due to coalescence occurs.

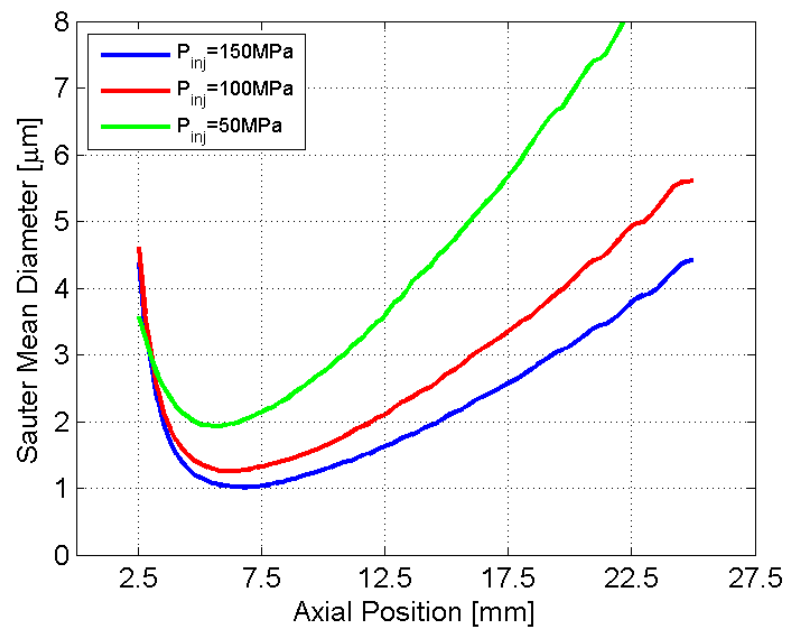

Figure 12. Computed SMD profiles for different injection pressures at $1.5 \mathrm{~ms}$ after SOI

In the end, SMD predictions for the different back pressures are presented in Figure 13. The lowest back pressure condition shows a reduced atomization rate, as previously suggested, of the droplet size. Also, one can observe that the position at which the minimum droplet size occurs, is located further downstream of the nozzle exit in comparison with the nominal operation point and finally, there is a minimized coalescence effect with the axial distance due to the reduced ambient density environment. 


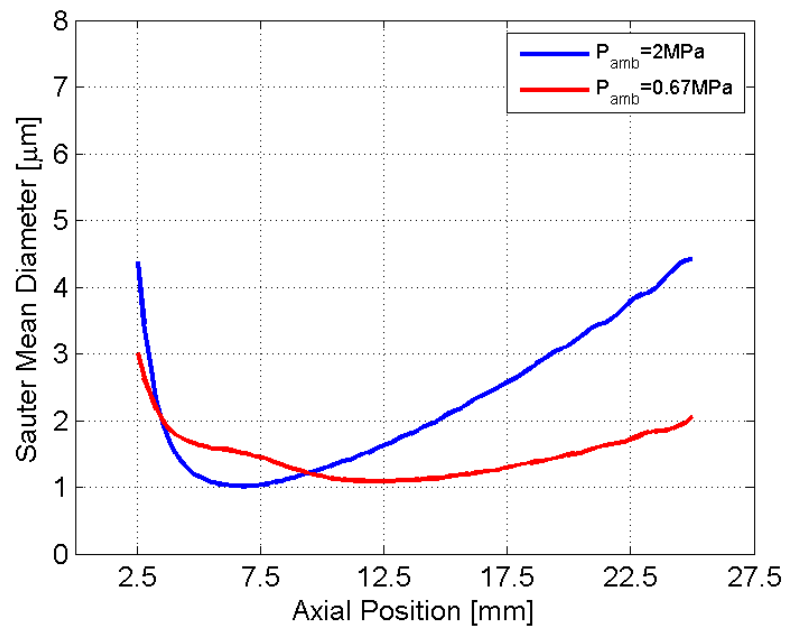

Figure 13. Computed SMD profiles for different back pressures at $1.5 \mathrm{~ms}$ after SOI

\section{Conclusions}

Though the interfacial evolution model had, in the past, been adjusted to better match experimental data, such adjustments had not been based on interfacial area. The fact that both the model and experiments are directly reporting interfacial area allows "apples to apples” comparison.

Because a new validation concept was employed, past suggestions of the parameters were not considered valid. Instead, a baseline condition was used to set two unknown parameters in the interfacial evolution model. Without further adjustment, the model was able to fairly accurately predict the interfacial behavior for a range of injection pressures.

At higher downstream pressure, where the ambient density was high and the mixing-limited hypothesis [15] holds, the accuracy was also good. However, the predictions of the model were slightly less accurate at a lower downstream pressure, where the mixing-limited hypothesis is less applicable.

The results suggest that the $\Sigma$-Y model provides good predictive power in the dense spray core, especially when the operating conditions correspond to typical diesel conditions, where the spray evolution is mixing-limited.

The validation using USAXS was particularly valuable, because neither the experiments nor the models relied on the assumption of distinct droplets. Only further downstream, when primary atomization is complete, is such an assumption appropriate.

In order to more fully gage the predictive power of this model, future studies will consider other injector configurations and conditions. For the model to prove fully predictive, further testing must show that with other fuel injectors, the model can be used without further adjustment.

\section{References}

1. Gorokhovski, M. and Herrmann, M., "Modeling primary atomization.” Annu. Rev. Fluid Mech. 40 (2008): 343-366.

Page 8 of 9
2. Linne, M., Paciaroni, M., Hall, T. and Parker, T., "Ballistic imaging of the near field in a diesel spray." Experiments in fluids 40, no. 6 (2006): 836-846.

3. Blokkeel, G., Barbeau, B. and Borghi, R., “A 3d Eulerian model to improve the primary breakup of atomizing jet." Atomization and Sprays 11 (2001): 619-642.

4. Arienti, M. and Sussman, M., "A Numerical Study of the Thermal Transient in High-Pressure Diesel Injection.” International Journal of Multiphase Flow 88 (2017): 205-221.

5. Trask, N. A., "Implementation of an Eulerian atomization model to characterize primary spray formation." Masters Theses (2010): 434.

6. Lacaze, G., Misdariis, A., Ruiz, A. and Oefelein, J. C., "Analysis of high-pressure Diesel fuel injection processes using LES with real-fluid thermodynamics and transport." Proceedings of the Combustion Institute 35, no. 2 (2015): 16031611.

7. Harlow F. H., Amsden A. A., "Numerical calculation of multiphase fluid flow.” J. Comput Phys 17 (1975): 19-52.

8. Iyer V. A., Abraham J., Magi V., "Exploring injected droplet size effects on steady liquid penetration in a diesel spray with a two-fluid model”. Int J Heat Mass Transf 45 (2002): 519-531.

9. Vujanovic M., Petranovic Z., Edelbauer W., Baleta J., Duic N., "Numerical modelling of diesel spray using the Eulerian multiphase approach.” Energy Conversion and Management. 104 (2015) 160-169.

10. Garcia-Oliver, J. M., Pastor, J. M., Pandal, A., Trask, N., Baldwin, E. and Schmidt, D. P., "Diesel spray CFD simulations based on the $\Sigma$-Y Eulerian Atomization model." Atomization and Sprays 23, no. 1 (2013).

11. Demoulin, F.-X., Beau, P. A., Blokkeel, G., Mura, A. and Borghi, R., "A new model for turbulent flows with large density fluctuations: Application to liquid atomization.” Atomization and Sprays 17, no. 4 (2007).

12. Demoulin, F.-X., Reveillon, J., Duret, B., Bouali, Z., Desjonqueres, P. and Menard, T., "Toward using direct numerical simulation to improve primary break-up modeling." Atomization and Sprays 23, no. 11 (2013).

13. Xue, Q., Battistoni, M., Powell, C. F., Longman, D. E., Quan, S. P., Pomraning, E., Senecal, P. K., Schmidt, D. P. and Som, S., "An Eulerian CFD model and X-ray radiography for coupled nozzle flow and spray in internal combustion engines." International Journal of Multiphase Flow 70 (2015): 77-88.

14. Desantes, J. M., Garcia-Oliver, J. M., Pastor, J. M. and Pandal, A., "A comparison of Diesel sprays CFD modeling approaches: DDM vs $\Sigma$-Y Eulerian Atomization model.” Atomization and Sprays 26, no. 7 (2016).

15. Pandal, A., Pastor, J. M., García-Oliver, J. M., Baldwin, E. and Schmidt, D. P., "A consistent, scalable model for Eulerian spray modeling.” International Journal of Multiphase Flow 83 (2016): 162-171.

16. Pischke, P., Kneer, R. and Schmidt, D. P., “A comparative validation of concepts for collision algorithms for stochastic particle tracking.” Computers \& Fluids 113 (2015): 77-86.

17. Lebas, R., Menard, T., Beau, P.A., Berlemont, A. and Demoulin, F.-X., "Numerical simulation of primary break-up and atomization: DNS and modelling study", Int. J. Multiphase Flow, 35 (2009), pp. 247-260

18. Siebers, D.L., "Liquid-phase fuel penetration in diesel sprays based on mixing-limited vaporization”, Trans. SAE, 108 (1999), pp. 703-728

19. Narayanan, T., "Synchrotron Small-angle X-ray Scattering." In Soft Matter Characterization, edited by Redouane Borsali and Robert Pecora, 899-952. Springer Netherlands, 2008. 
20. Ilavsky, J., Jemian, P. R., Allen, A. J., Zhang, F. et al., "Ultrasmall-angle X-ray scattering at the Advanced Photon Source”. J. Appl. Cryst. 42(3):469-479, doi:10.1107/S0021889809008802.

21. Ilavsky, J. and Jemian, P. R., "Irena: Tool Suite for Modeling and Analysis of Small-angle Scattering." J. Appl. Cryst. 42(2):347-353, doi:10.1107/S0021889809002222.

22. Vallet, A., Burluka, A. and Borghi, R., "Development of a Eulerian Model for the Atomization of a Liquid Jet." Atomization and Sprays 11 (2001): 619-642

23. Reid, R., Prausnitz, J. and Poling, B., "The Properties of Gases and Liquids.” McGraw-Hill, 1987

24. Trask, N., Schmidt, D. P., Lightfoot, M. and Danczyk, S., "Compressible Modeling of the Internal Flow in a Gas-Centered Swirl-Coaxial Fuel Injector.” Journal of Propulsion and Power 28, no. 4 (2012): 685-693

25. Ishii, M., "Thermofluid Dynamics of Two-phase Flows." Eyrolles, Paris, France, 1975

26. Delhaye, J., Giot, M. and Riethmuller, M., "Thermohydraulics of two-phase systems for industrial design and nuclear engineering.” Hemisphere Pub. Corp., 1981

27. Candel, S. and Poinsot, T., "Flame Stretch and the Balance Equation for the Flame Area." Combustion Science and Technology 70 (1990): 1-15

28. Marble, F. and Broadwell, J., "The Coherent Flame Model for Turbulence Chemical Reactions.” Purdue University, Technical report, Project Squid Headquarters. Chaffee Hall, Purdue University, West Lafayette, Indiana, 1977

29. Vallet, A. and Borghi, R., "Modélisation Eulerienne de l'atomisation d'un jet liquide.” C.R. Acad. Sci, Paris 327 (1999): 1015-1020

30. Beheshti, N., Burluka, A. and Fairweather, M., "Assessment of $\Sigma$-Y liq model predictions for air-assisted atomisation." Theoretical and Computational Fluid Dynamics 21 (2007): 381397

31. Belhadef, A., Vallet, A., Amielh, M. and Anselmet, F., "Pressure-swirl atomization: Modeling and experimental approaches". International Journal of Multiphase Flow 39 (2012): 13 - 20

32. Wang, Y., Lee, W., Reitz, R. and Diwakar, R., "Numerical Simulation of Diesel Sprays Using an Eulerian-Lagrangian Spray and Atomization (ELSA) Model Coupled with Nozzle Flow”. SAE Technical Paper2011-01-0386, 2011,

33. Wang, Y., Grover, R., Schmidt, D. P., Diwakar, R. and Kuo, T.W., "Application of Interface Area Density Modeling to Define Spray Plume Boundary.” ILASS Paper, 2015

34. Pope, S. B., “Turbulent Flows.” Cambridge University Press, 2000

35. Beau, P., Funk, M., Lebas, R. and Demoulin, F.-X., “Applying Quasi-Multiphase Model to Simulate Atomization Processes in Diesel Engines: Modeling of the Slip Velocity.” SAE Technical Paper 2005-01-0220, 2005

36. Desantes, J. M., García-Oliver, J. M., Pastor, J. M., Pandal, A., Baldwin, E. and Schmidt, D. P., "Coupled/decoupled spray simulation comparison of the ECN spray a condition with the $\Sigma$ Y Eulerian atomization model." International Journal of Multiphase Flow 80 (2016): 89-99

37. Kastengren, A., Ilavsky, J., Viera, J., Payri, R., Duke, D., Swantek, A., Tilocco, F., Sovis, N. and Powell, C., "Measurements of Droplet Size in Shear-Driven Atomization Using Ultra-Small Angle X-Ray Scattering". International Journal of Multiphase Flow, 2016 (under review)

\section{Contact Information}

Adrian Pandal

adrianpandal26@gmail.com

pandaladrian@uniovi.es

\section{Acknowledgments}

Authors acknowledge that part of this work was partially funded by the Spanish Ministry of Economy and Competitiveness in the frame of the COMEFF (TRA2014-59483-R) project.

\section{Definitions/Abbreviations}

\begin{tabular}{|c|c|}
\hline CFD & $\begin{array}{l}\text { Computational } \\
\text { Dynamics }\end{array}$ \\
\hline DNS & Direct Numerical Simulation \\
\hline ELSA & $\begin{array}{l}\text { Eulerian-Lagrangian } \\
\text { and Atomization }\end{array}$ \\
\hline HBT & Hankinson-Brobst-Thomson \\
\hline LES & Large-Eddy Simulation \\
\hline RANS & $\begin{array}{l}\text { Reynolds-Averaged Navier- } \\
\text { Stokes }\end{array}$ \\
\hline SMD & Sauter-Mean-Diameter \\
\hline USAXS & $\begin{array}{l}\text { Ultra-Small-Angle } \\
\text { Scattering }\end{array}$ \\
\hline
\end{tabular}

